\title{
LE RÔLE DES INTERNAUTES DANS LA CO-CONSTRUCTION DE LA CROYANCE EN MICHAEL JACKSON : DU KING OF POP À WACKO JACKO
}

\begin{abstract}
Amélie Dalmazzo ${ }^{1}$
Michael Jackson, pur produit de la culture populaire, mass-médiatique et transnationale, a su engager la croyance de communautés et d'individus divers depuis plus de 40 ans. Cette figure est le fruit d'une co-construction : les médias, l'industrie culturelle, et les publics ont chacun produit des récits, des imageries, qui se sont influencées réciproquement et ont soutenu l'adhésion des publics. Les fans notamment, se sont servis d'Internet pour s'approprier le héros et diffuser leur vision du chanteur. Empruntant à de nombreuses généalogies, ils ont créé une imagerie glorifiante, qui a amplement densifié sa mythologie et qui a façonné en grande partie les contours du King of Pop. Produisant et diffusant des contenus eux-mêmes appropriables par les publics, ils sont devenus de véritables prescripteurs de sens. Ils ont renforcé le pouvoir d'attraction de Michael Jackson en participant pleinement à l'élaboration de son charisme. Les détracteurs du chanteur, quant à eux, ont également eu recours aux nouvelles technologies : par la création de sites web anti-Michael Jackson ou par la production et la diffusion de caricatures et de détournements d'image, ils ont tenté de discréditer la star
\end{abstract}

1 Amélie Dalmazzo est Docteure en science de l'information et de la communication et sémiologue des médias.

Recherches en communication, $\mathrm{n}^{\circ} 38$ (2012). 
et d'influencer notre vision du personnage. Représentant le chanteur sous les traits de Wacko Jacko (« Jacko le dément ») - une créature crépusculaire et repoussante - ils ont fabriqué, en association avec les médias traditionnels, la monstruosité de Michael Jackson. Ces productions amateurs, remplies d'affects positifs ou négatifs, ont accru le polymorphisme du chanteur et lui ont conféré une ambivalence fascinante apte à encourager l'identification et la projection du plus grand nombre.

Plus qu'un être fait de chair et de sang, Michael Jackson est un véritable récit interactif façonné par l'imaginaire collectif. En écrivant de concert son histoire, l'industrie culturelle, les médias et les publics ont élaboré une créature capable d'incarner tous les paradoxes, de générer de nombreuses communautés, et de répondre aux besoins identitaires des publics. Mieux encore, figure ambivalente, à la fois charismatique et monstrueuse, Michael Jackson a su exprimer une part de l'inconscient de chacun et s'offrir comme un fabuleux support de croyance. Par « croyance », et comme Michel De Certeau (1987), je ne considère pas « les contenus » de la croyance, mais l'investissement d'un sujet pour une proposition qui lui est faite. Dans une telle conception, il s'agit de désigner la nature et la quantité d'affects projetés par un individu ou une communauté sur un « objet $^{1}$ » tel qu'il est entendu par la psychanalyse freudienne, c'est-à-dire sur un réceptacle d'identifications protéiformes investi comme une surface projective pouvant parfois tenir lieu « d'Idéal du $\mathrm{Moi}^{2}$ » personnel ou collectif ${ }^{3}$ (Freud, 1918 ; Kaës, 1998).

Cependant, considérant autant les affects positifs que négatifs, j'estime que la croyance s'exprime tant par le biais d'une adhésion partisane que d'une passion haineuse : en effet, certains croyants souvent considérés comme " fans " - éprouvent le désir impérieux de s'approprier l'objet, tandis que d'autres - les « fans-anti » et les

1 La notion d' « objet » est envisagée « en tant que corrélatif de la pulsion : il est ce en quoi et par quoi celle-ci cherche à atteindre son but, à savoir un certain type de satisfaction. Il peut s'agir d'une personne [...], d'un objet réel ou d'un objet fantasmatique » (Laplanche \& Pontalis, 2002, p. 290).

2 Pour Freud, cet idéal du moi est une instance intrapsychique différenciée qui " constitue un modèle auquel le sujet cherche à se conformer ». (Laplanche \& Pontalis, 2002, p. 184).

3 S'inspirant de la conception de la foule que Freud développe en 1918, R. Kaës (1998), considère que les membres d'un groupe modifient leur Idéal du Moi personnel dans le sens d'un consensus, afin de donner naissance à un idéal collectif. 
« fanopathes ${ }^{1}$ » (Dalmazzo, 2009) - veulent à tout prix le mettre à distance. Michael Jackson catalyse d'ailleurs autant 1'admiration que la critique. Il s'incarne à travers trois grands archétypes : King of Pop, personnification de la toute puissance et de l'immortalité ; Wacko Jacko, figure monstrueuse et démente ; et Bambi, innocent candide au regard de biche. Chacun de ces archétypes, soutenus par des communautés de croyance spécifiques - dont j'étudierai ici quelques cas emblématiques mobilisent leurs propres publics, récits et modalités d'adhésion.

L'analyse sémiologique des récits ${ }^{2}$ amateurs produits par les internautes avant la mort de la star me permettra de rendre compte de l'implication des publics dans la co-construction de la croyance en Michael Jackson, de son « charisme médiatique » et de sa « monstruosité médiatique » (Dalmazzo, 2009), des formes que ceux-ci prennent par leur construction artificielle par des producteurs d'image, formes qui sont consommées et appropriées massivement via les médias et les produits culturels. Images mises en scène et séparées de la présence du corps, données à un public lors d'une interaction médiatique, elles sont des « construits langagiers » constitués d'un ensemble composite de récits aptes à encourager la croyance et à induire la fascination.

Il faut préciser que le charisme et la monstruosité du chanteur sont également de type « transculturels ${ }^{3}$ » (Dalmazzo, 2009) : Michael Jackson est une star internationale capable de séduire, de repousser, et d'encourager la croyance chez des publics aux profils socioculturels hétérogènes, possédant pourtant des codes et langages divers. Ce constat m'invite à penser que les récits co-construisant sa mythologie, véhiculés par des médias transnationaux, reposent sur des référentiels

1 Fans vivant leur « fanitude » comme un facteur déstructurant (Dalmazzo, 2009).

2 Dans cet article, le terme " récit » désigne l'ensemble des images, textes, et enregistrements sonores ou visuels qui sont mis à disposition des publics via les médias et les NTIC. Selon moi, une image ou une chanson, par exemple, forment autant un récit qu'un texte scriptural.

3 Dans ma conception, le « charisme transculturel » ne s'adresse jamais à un seul groupe social, ou à une seule communauté culturelle en attente de leader. Il parvient au contraire à engendrer de nouvelles communautés : des « communautés de charisme » (Dalmazzo, 2009). Dans ce cas, la figure charismatique ne répond pas seulement aux désirs et besoins d'un groupe spécifique, mais avant tout aux attentes des publics sur le plan individuel (besoins identitaires sociaux ou psychiques, Camilleri et al., 1997). 
internationaux et renferment des significations évocatrices capables d'entrer en résonance avec l'inconscient de chacun. Comme Tisseron (1997), je considère les apports féconds d'une rencontre entre la psychanalyse et l'analyse de l'image ou des mythes. Il s'agira alors pour moi, à l'instar d'Assoun ou de Fromm, d'explorer " le bord inconscient du processus social ${ }^{1} »$ (Assoun, 1990, p. 88) que je décrirai plutôt que de déterminer les significations anthropologiques propres à la réception ${ }^{2}$ des récits par des publics socioculturellement définis.

En premier, je décrirai le processus de co-construction de la croyance en la star. En second, j'illustrerai ce mécanisme à l'aide d'une page Web consacrée à l'évolution des représentations du chanteur sur une période de 25 ans, en montrant comment il passe progressivement de figure charismatique à figure monstrueuse. En troisième, je démontrerai qu'au terme de ce long processus, Michael Jackson s'est établi comme une figure mythique évoquant quelques héros ancestraux. Enfin, je décrirai la façon dont les internautes tentent, après la mort du chanteur, d'institutionnaliser la croyance en son mythe.

\section{La co-construction de la croyance en Michael Jackson}

Le modèle proposé par Philippe Marion (1997) sur « l'intermédialité » est très utile pour expliquer la manière dont la croyance en Michael Jackson s'est construite. Pour cet auteur, l'intermédialité désigne l'ensemble des interactions médiatiques qui participent à la production du sens : elle s'intéresse à la relation établie entre différents supports médiatiques, dès lors qu'ils deviennent les porteurs d'un seul et même récit, qu'ils construisent en synergie.

1 Selon ces auteurs, les phénomènes collectifs peuvent être analysés au regard des fonctionnements psychiques et des motivations inconscientes des individus et des groupes. Erich Fromm est, avec Theodor W. Adorno et Herbert Marcuse, l'un des premiers représentants de l'École de Francfort. Pour l'essentiel son approche a consisté à greffer la thèse freudienne sur la réalité sociale de l'après-guerre. Il est notamment connu pour son étude du nazisme en tant que phénomène psychologique.

2 Selon moi, le charisme résulte d'un processus interactif et communicationnel qui implique autant le producteur d'image que le récepteur. Le récepteur participe ainsi activement à la co-construction de la personnalité : d'une part, par l'intermédiaire du mécanisme de projection, il décode activement les images et récits qui lui sont donnés à consommer; d'autre part, il participe lui-même à l'écriture du mythe en produisant à son tour des récits et des images amateurs, qui sont eux-mêmes diffusés et appropriés par les publics. 
Selon lui, les récits médiatiques contemporains sont caractérisés par leur portée «transmédiatique » et « intergénérique » : ils se déclinent sur de nombreux supports et adaptent leurs formes à divers genres. " Le médiatique, lorsqu'il prend la forme d'une dissémination organisée, peut alors se concevoir comme une mise en réseau [des récits]. » (Marion, 1997, p. 70) Ainsi le mythe " Michael Jackson » s'est constitué de manière kaléidoscopique à travers l'empilement de récits fragmentaires et épisodiques recyclés en permanence, et innervant l'ensemble du champ médiatique et culturel : CD, DVD, livres (romans et biographies), films (Moonwalker ${ }^{1}$, This is $i t^{2}$ ), dessins animés (Jackson 5 Cartoon $^{3}$ ), bandes dessinées, ou encore jeux vidéos (Moonwalker ${ }^{4}$ ), sagas médiatiques, détournements d'images, récits amateurs, parc d'attraction (Captain Eo $o^{5}$ ), déguisements (Wacko jacko Mask)... Ces récits et imageries qui s'influencent réciproquement sont produits par trois acteurs : l'industrie culturelle (incluant l'artiste), les médias et les publics.

En premier lieu, les industries culturelles - soit l'industrie musicale en interaction constante avec l'artiste et les sociétés d'édition musicale qui l'ont soutenu - définissent le style du chanteur, son univers et son identité de marque. À travers les clips, textes de chansons, photos promotionnelles, et toute l'imagerie officielle, elles élaborent les contours du King of Pop, un magicien omnipotent, polymorphe, et immortel appartenant à une lignée prestigieuse.

En second lieu, les médias récupèrent cette première imagerie pour créer d'autres récits et déformer les contours du personnage. Par l'intermédiaire des news, commentaires, et sagas médiatiques qu'ils produisent, ou en inventant et relayant de multiples rumeurs sur le chanteur, ils initient une seconde imagerie. Ainsi en 1986, ils donnent naissance à Wacko Jacko, le double inversé du King of Pop, qui apparaît pour la première fois à la Une du tabloïd britannique The Sun :

1 Film réalisé par J. Kramer et C. Chilvers, décembre 1988.

2 Documentaire post mortem réalisé par K. Ortega, octobre 2009.

3 Série de dessins animés dont les Jackson Five sont les héros, diffusée dans les années 1970 aux États-Unis et dans les années 1990 en France.

4 Dans les années 1980, le jeu vidéo Moonwalker (pour consoles Megadrive) reprend le scénario du film.

5 Film en trois dimensions réalisé par Fr. F. Coppola (1987), diffusé dans les parcs Disneyland. 
photographié dans un caisson à oxygène qu'il a offert à un hôpital, le chanteur fait la couverture. Un gros titre annonce : «Wacko Jacko. Son lit est un caisson à oxygène coûtant $85.000 £$ ». Une légende ajoute : " Enfermé dans un caisson de verre, Michael Jackson respire un air enrichi en oxygène. Il espère ainsi pouvoir vivre jusqu'à 150 ans ». En tant que véritables instances de légitimation, les médias crédibilisent l'exceptionnalité de Michael Jackson au point de le transformer peu à peu en une créature surréelle s'affranchissant de toutes les normes. Ce faisant, ils renforcent la croyance en son charisme : semblable à un surhomme, il paraît capable de défier la mort. D'emblée, les fantasmes inconscients de toute-puissance que certains nourrissent pour eux-mêmes s'en trouvent stimulés : si Michael Jackson est capable de réaliser l'impossible, alors peut être qu'en s'identifiant à lui les publics pourront, à leur tour, accomplir des exploits. Les figures charismatiques parviennent à faire croire à leurs publics qu'elles ont des capacités supérieures et qu'en les imitant, ils atteindront l'idéal qu'ils convoitent (en l'occurrence ici la toute puissance narcissique : l'immortalité, l'omnipotence, la désirabilité) (Dalmazzo, 2009). C'est pour cela qu'elles sont jugées exceptionnelles.

Cependant, simultanément, les médias accentuent l'étrangeté du chanteur et font ainsi naître chez d'autres un sentiment d'inquiétude dérangeant. Le chanteur, qui incarne les désirs inconscients des premiers, figure simultanément les peurs inconscientes des autres (en l'occurrence, je l'expliciterai par la suite, le chanteur figure l'aliénation narcissique qui menace tous ceux qui croient en l'illusion d'une toute puissance possible).

Les publics enfin s'approprient les récits de l'industrie et ceux des médias pour y projeter leur propre fantasmatique et imaginaire. Par l'intermédiaire de la rumeur mais aussi de leurs caricatures et détournements d'images - tantôt positifs et tantôt négatifs - ils façonnent une troisième imagerie qui pourra elle-même être partagée et appropriée par les communautés de croyance. Les rumeurs les plus folles ajoutent ainsi peu à peu à la puissance évocatrice du personnage : il aurait, par exemple, une réelle capacité de métamorphose. Pour preuve, lui et ses sœurs ne seraient qu'une seule et même personne ${ }^{1}$ ! La

1 Rumeur circulant au début des années 1990. 
participation active des publics au processus de mythisation, renforcée par la démocratisation des NTIC et particulièrement celle d'Internet, accroît la dimension cathartique du mythe, et facilite son appropriation en tant que surface projective et objet de croyance.

\section{La construction d'un surhomme}

Une page Web nommée A Photographic History of Michael Jackson's Face ${ }^{1}$ (Figure 1), hébergée depuis 2003 et réalisée par l'internaute Rahni, illustre ce processus interactifde co-construction. Une série de comparaisons proposée par ce dernier révèle les appropriations successives de l'image du chanteur par les publics entre 1979 et 2004, telles qu'elles ont été conditionnées par les transformations physiques du chanteur, les effets de sa communication et 1'évolution des récits médiatiques produits à son sujet.

La première comparaison de cet article confronte le Michael Jackson de 1985 au Dr. Frank'n Further, héros du film The Rocky Horror Picture Show ${ }^{2}$ et savant fou ayant donné vie à une créature. Cette association révèle qu'à l'époque la plupart des médias et des publics produisent des images et des récits élogieux au sujet du chanteur. Les effets de sa communication officielle se font sentir car, en 1984, à l'occasion d'un clip qui lui apportera une renommée internationale incomparable ${ }^{3}$, Michael Jackson est, à l'instar de Frank'n Further, un créateur de génie ayant inventé un personnage fantastique : le zombie de Thriller ${ }^{4}$.

1 www.anomalies-unlimited.com/Jackson.html

2 Film musical anglo-américain sorti en 1975. Adaptation du réalisateur australien Jim Sharman de la comédie musicale éponyme écrite par Richard O’Brien.

3 L'album Thriller sorti en 1982 est encore à ce jour l'album solo le plus vendu de tous les temps : 104 millions d'exemplaires en janvier 2008 (Guinness Book des records, 2008).

4 Clip d'environ 14 minutes réalisé par John Landis. 


\begin{tabular}{|c|c|c|}
\hline \multicolumn{3}{|c|}{$\begin{array}{l}\text { A Photographic History of Michael Jackson's Face } \\
\text { With blithering, yet witty commentary }\end{array}$} \\
\hline & \multicolumn{2}{|c|}{$\begin{array}{l}\text { Hard to believe - this was Michael Jackson. He was born August 28, } 1958 \text { - one of } 9 \text { kids. } \\
\text { His father reportedly nicknamed him "Big Nose". } \\
\text { Mike was born a cute African-American guy. "Normal", if you will, and very talented. } \\
\text { Despite the current, sad stories about his lonely, sad childhood, Mike grew up surrounded } \\
\text { by famous people and an adoring public. At age } 5 \text {, Mike and his brothers were the } \\
\text { amazing 'Jackson 5'. They played locally, then in New York and Philly. They were } \\
\text { "discovered" by Gladys Knight and pianist Billy Taylor at the famous Apollo Theater in } \\
\text { Harlem. By age 11, Mike was a Superstar. At age } 13 \text { he went solo and had his first \#1 hit } \\
\text { at } 14 \text { with "Ben" (a touching love song to a rat). Who knew he'd get addicted to plastic } \\
\text { surgery, face accusations of child molestation and end up America's Most Famous } \\
\text { Sideshow? }\end{array}$} \\
\hline 1984 age & \multicolumn{2}{|c|}{$\begin{array}{l}\text { Mike gets his nose slightly narrowed and his eyebrows shaped. This was his "Thriller" Era and he was } \\
\text { smokin'. People did notice this facial change and commented on it - guys just didn't do this back then. } \\
\text { Some in the Black community made comments about him having a problem with his African-American } \\
\text { looks and making his nose more "White". He was cute as hell, though. Oh, baby. He gave coherent } \\
\text { interviews. He had a cute sense of humor and was seen on TV doing other things besides whining, } \\
\text { faking tears and defending legal charges. He didn't wear a face mask in public. He smiled a big, } \\
\text { infectious smile. He was humble and grateful for his fame and his fans' appreciation. He made hit after } \\
\text { hit, celebrated music videos one after another, sealed obscenely huge record sales and contracts. He } \\
\text { had unprecedented \$ponsorship deals with Pepsi, and LA Gear Sportwear. People stood in line at 1AM to } \\
\text { purchase "Thriller" when it came out, even though the store didn't open until } 9 \text { AM. }\end{array}$} \\
\hline 198 & $\begin{array}{l}\text { - The shark music from "Jaws" starts softly in the background... } \\
\text { Another nose job to narrow things and permanent eyeliner tattooed around his } \\
\text { eyes. Ouch! Is that lipstick?! Hell, it's the } 80 \text { s - it's allowed. During that time he } \\
\text { had an army of spin doctors, lawyers, bodyguards, agents, minions, PR } \\
\text { magicians, attendants, and managers all making sure he no one had a clue about } \\
\text { his personal life but what did we care? He was doing amazing, selfless things - } \\
\text { contributing to children's charities and starting his own "Heal The World } \\
\text { Foundation"; cowriting the famous "We Are The World" song to help African } \\
\text { famine victims. He was given the Heritage Award and praised by Queen Liz, } \\
\text { President Reagan and others. Mike was everywhere, giving as much as he got } \\
\text { and letting us all know how blessed he was. There was no one who wasn't } \\
\text { impressed and didn't sprain an ankle trying to imitate his "Moon Walk" in their } \\
\text { living room. }\end{array}$ & $\begin{array}{l}\text { Almost, but not quite } \\
\text { The Rock Horror } \\
\text { Show }\end{array}$ \\
\hline & $\begin{array}{l}\text { - The Sigourney Weaver in "Ghostbusters" Stage - The beginning of } \\
\text { the end. } \\
\text { OK, people and the press are really talking now. Gasps are audibly heard. He } \\
\text { gets his nose done again, and, in a move that will forever baffle the world, } \\
\text { neglects to sue the bastard who botched the surgery job on him. He suddenly has } \\
\text { cheek bones. In a mere year and a half his skin's gone from beautiful cocoa } \\
\text { bronze to fish belly white. He first denies this, then blames it on the medical } \\
\text { condition Vitiligo which causes people of color to develop light patches of skin } \\
\text { that ladk pigment. Well he doesn't say this, his "people" say this. Mike ain't } \\
\text { saying a thing which is odd considering the good he could do to bring this little } \\
\text { understood condition to public light. Rumors abound that he's been allegedly } \\
\text { taking female hormones (note the clever use of the word "allegedly" to avoid a } \\
\text { law suit) to remove facial hair and keep that voice of his at the } 12 \text { year old boy } \\
\text { pitch. He's talking in a Marilyn Monroe Little Girl Whisper. He's started the Spin of } \\
\text { the misunderstood, picked-upon Victim instead of an increasingly weird } 30 \text { year } \\
\text { old man. He's creepy. People are making jokes that only in America can you be } \\
\text { bom a black man and end up a white woman. Talented or no, the fact is we're } \\
\text { realizing that Michael Has Issues. }\end{array}$ & 1ey \\
\hline & \begin{tabular}{|l} 
- The "LaToilet" stage \\
In an insult to transvestite men everywhere - who can look pretty damn good in \\
a dress and makeup and can project alluring female charm - when Mike does this,
\end{tabular} & \\
\hline
\end{tabular}

FIGURE 1 
La suite de l'article, qui porte sur le Michael Jackson de 1987 à 1993, montre que la croyance en son mythe se renforce progressivement sous l'effet de l'interaction permanente entre les trois acteurs de la co-construction : les frontières entre le créateur et la créature s'estompent pour façonner les contours d'un véritable surhomme capable de toutes les extravagances et de tous les exploits. Selon Rahni, 1987 marque un véritable tournant : il assimile ainsi le chanteur au personnage interprété par Sigourney Weaver dans le film SOS Fantômes ${ }^{1}$. L'actrice y incarne une femme rangée qui se transforme en tigresse redoutable sous l'emprise d'un esprit diabolique. Cette comparaison suggère que la créature enfantée par Michael Jackson - son personnage public, alors nommé King of Pop - a pris possession de lui, à la manière du fantôme qui hante l'héroïne de SOS Fantômes. Elle révèle la tendance des publics à assimiler le Michael Jackson réel au personnage imaginaire qu'il interprète. C'est d'ailleurs en 1986 qu'apparaît Wacko Jacko : l'excentricité du personnage fictionnel interprété par la star dans ses vidéos promotionnelles (clips, films de cinéma, bandes annonces, etc.) est dorénavant prise pour la réalité. Les médias, capables de légitimer toutes les rumeurs, dépeignent un Michael Jackson « réel » et intime semblable à l'étrange créature publique qu'il incarne. Il est utile de préciser que cette assimilation entre la personne et le personnage intervient au moment où l'album $B a d^{2}$ sort dans les bacs. Or à cette période, le chanteur joue plus que jamais de son ambiguïté : en interview, il s'exprime avec une voix enfantine (on l'appelle Bambi). À l'opposé, dans ses clips, il interprète un chef de gang et revendique son côté bad boy: habillé d'un pantalon de cuir clouté, il se déhanche de façon provocante. De même son apparence a particulièrement changé : sa peau s'est éclaircie, son look est plus androgyne que jamais... Cette ambivalence que cultive Michael Jackson, lui confère une dimension de plus en plus insaisissable qui autorise chacun à projeter en lui son propre imaginaire.

Cette tendance ne fera que s'accentuer avec la sortie en $1988 \mathrm{du}$ film Moonwalker, dans lequel Michael Jackson s'évertue à gommer les frontières entre faits réels, rumeurs, fiction et mythologie. Dans l'esprit des publics, l'exceptionnalité surhumaine du chanteur semble bien

1 Film américain réalisé par Ivan Reitman en 1984 (titre original : Ghostbusters).

2 Bad (1987) est le septième album solo de Michael Jackson et le troisième produit par Epics Records (Sony). 
« réelle » car le film Moonwalker est présenté comme une biographie retraçant le parcours artistique du chanteur depuis son plus jeune âge jusqu'à ses 33 ans, tandis qu'il est agrémenté de clips musicaux divers et qu'il s'appuie sur un scénario purement fictionnel (la star, dotée de super-pouvoirs - comme celui de pouvoir se transformer à loisir - sauve trois enfants des griffes d'un méchant truand). À ce titre, il est utile de souligner le fait que le chanteur, bien qu'il interprète un personnage fictif dans ce film et dans l'ensemble de ses clips, est systématiquement présenté comme le "vrai » Michael Jackson : c'est ainsi qu'il est toujours nommé dans les fictions dont il est le héros (voir par exemple Bad, Thriller ou plus récemment Men in Black). Cet amalgame entre la créature (le personnage) et le créateur(la personne)finitainsi par entrainer la confusion dans l'esprit des publics et semble renforcer leur croyance en la dimension surhumaine de Michael Jackson. Cette tendance est clairement illustrée dans l'article analysé : l'internaute Rahni établit un parallèle entre le Michael Jackson de 1993 (Figure 2) et l'héroöne de dessin animé Ms. Judy Jetson, une voyageuse intergalactique vivant dans un monde futuriste. Avec cette comparaison, l'auteur participe à l'iconisation du chanteur et encourage les publics à le percevoir comme un être fantastique possédant le pouvoir de traverser le temps et l'espace. À l'image des héros fictifs qu'il interprète dans ses clips, il serait capable de résister à la mort et de s'affranchir des lois physiques. Dans ma conception, les figures charismatiques incarnent les désirs inconscients de ceux qui les admirent ${ }^{1}$ (Dalmazzo, 2009). Or quels désirs pourraient être plus répandus que ceux d'acquérir l'omnipotence ou l'immortalité ? Si les publics se plaisent à croire en la mythologie de ce héros fantastique, c'est sans doute qu'une part d'eux-mêmes a envie de croire en la possibilité d'une toute-puissance humaine.

1 Ces désirs sont nombreux et varient en fonction des individus qui s'identifient à la figure charismatique. Cependant, je fais l'hypothèse que les figures au charisme transculturel (dont Michael Jackson fait partie) parviennent à incarner les désirs narcissiques des individus qu'elles fascinent : désirs d'omnipotence, désirs d'immortalité, désirs de complétude, désirs d'être désirable, etc. Ces désirs, qui figurent parmi les plus répandus et les plus transculturels (pour ne pas dire universels) sont inconscients et se retrouvent à un degré plus ou moins fort chez tous les individus (Dalmazzo, 2009). 


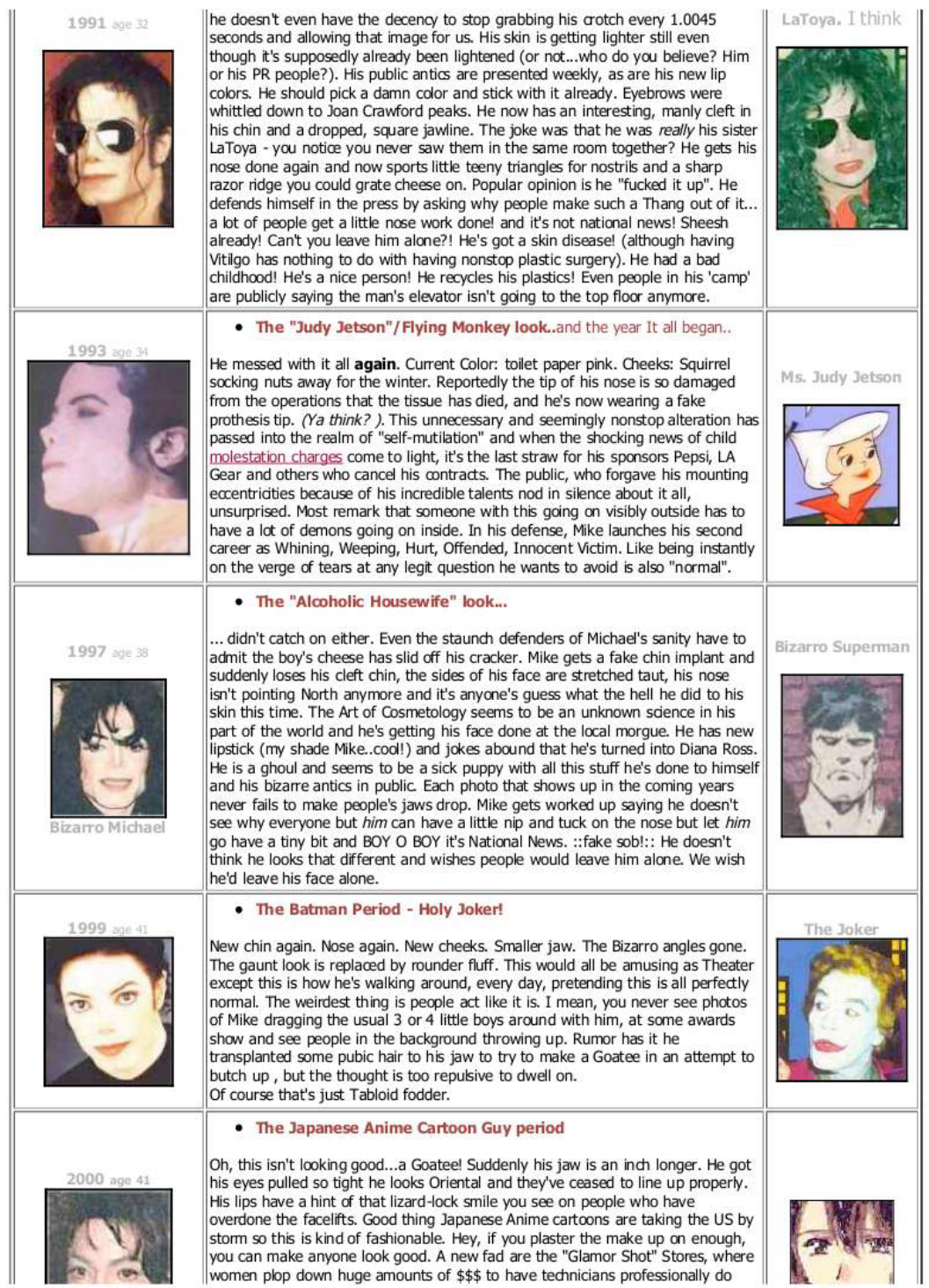

FIGURE 2 


\section{Du surhumain au non-humain}

Figure surhumaine, polymorphe et appropriable à loisir, Michael Jackson devient progressivement - sous l'effet conjoint des médias, des publics et de l'industrie culturelle - une pure abstraction, une image parfaitement malléable : chacun peut écrire un bout de son histoire, modifier les contours de son personnage et encourager dès lors de nouvelles modalités de croyance. La page Web (Figure 2) que j'analyse ici peut encore être prise en exemple, car elle décrit assez bien la manière dont le processus interactif de mythisation fait progressivement perdre toute humanité au chanteur. La comparaison de l'internaute amalgamant par exemple le Michael Jackson de l'an 2000 à un héros de manga japonais tout en images de synthèse, le révèle avec force : dès cette époque le chanteur n'est plus, dans l'esprit des publics, qu'un avatar numérique dont chacun pourrait définir les traits. Il serait apte à représenter, dans un au-delà virtuel, n'importe quelle individualité. De nouveau, cela encourage la fascination de ceux qui rêvent de trouver la complétude narcissique et de faire fusionner l'identité et l'altérité.

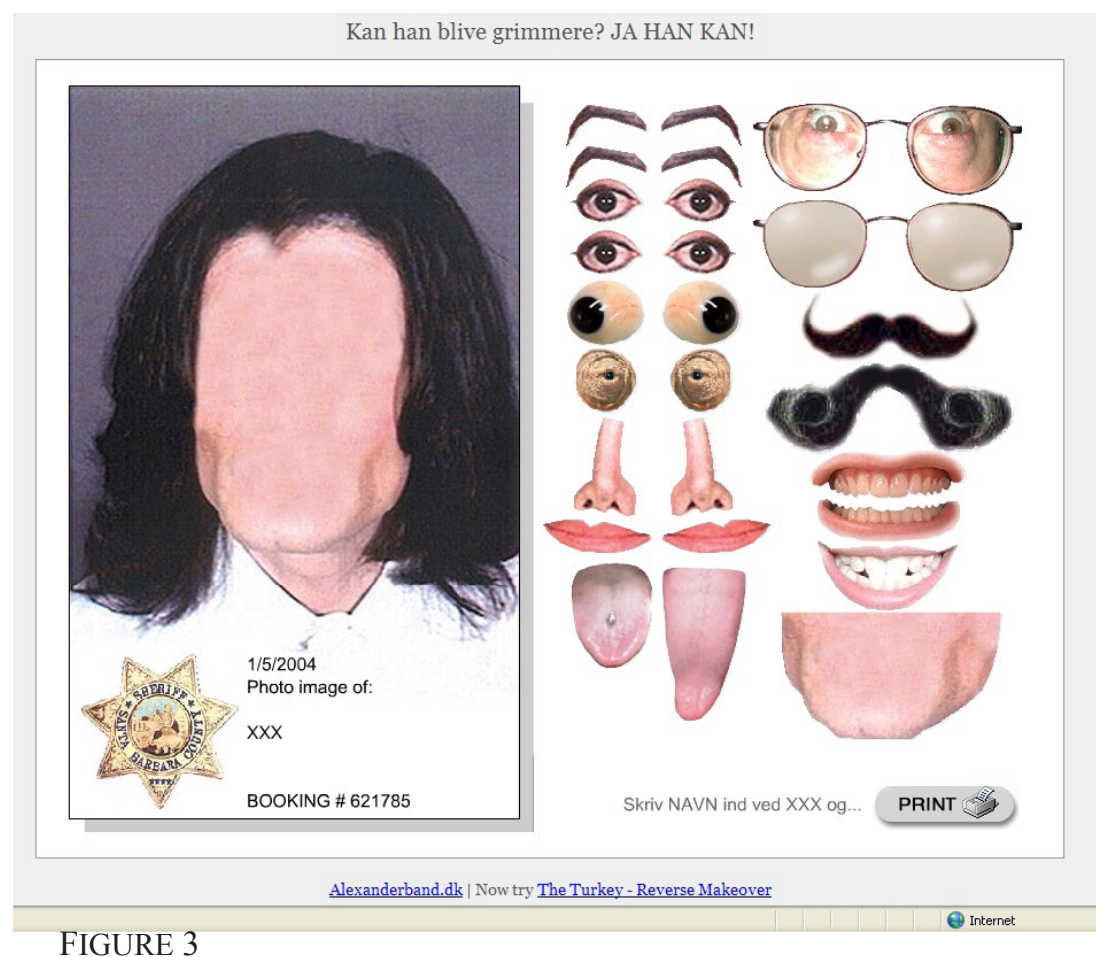


Une seconde page $\mathrm{Web}^{1}$ (Figure 3), qui est une application interactive diffusée en 2007, permet aux utilisateurs de constituer euxmêmes le visage de Michael Jackson. Cette représentation, qui accentue encore le polymorphisme du chanteur, renvoie au célèbre jouet Mister Potato Head, une poupée en forme de pomme de terre que les enfants peuvent agrémenter de nez, yeux, bouches, oreilles ou bras à leur convenance. Cette assimilation est relativement courante sur Internet. On la retrouve d'ailleurs sur la page de l'internaute Rahni, lorsqu'il compare le Michael Jackson de 2004 à cette poupée modulable éditée par Hasbro. Ou encore, on l'observe dans un photomontage ${ }^{2}$ (Figure 4) réalisé par un internaute anonyme et diffusé sur les réseaux sociaux au cours des années 2000 .

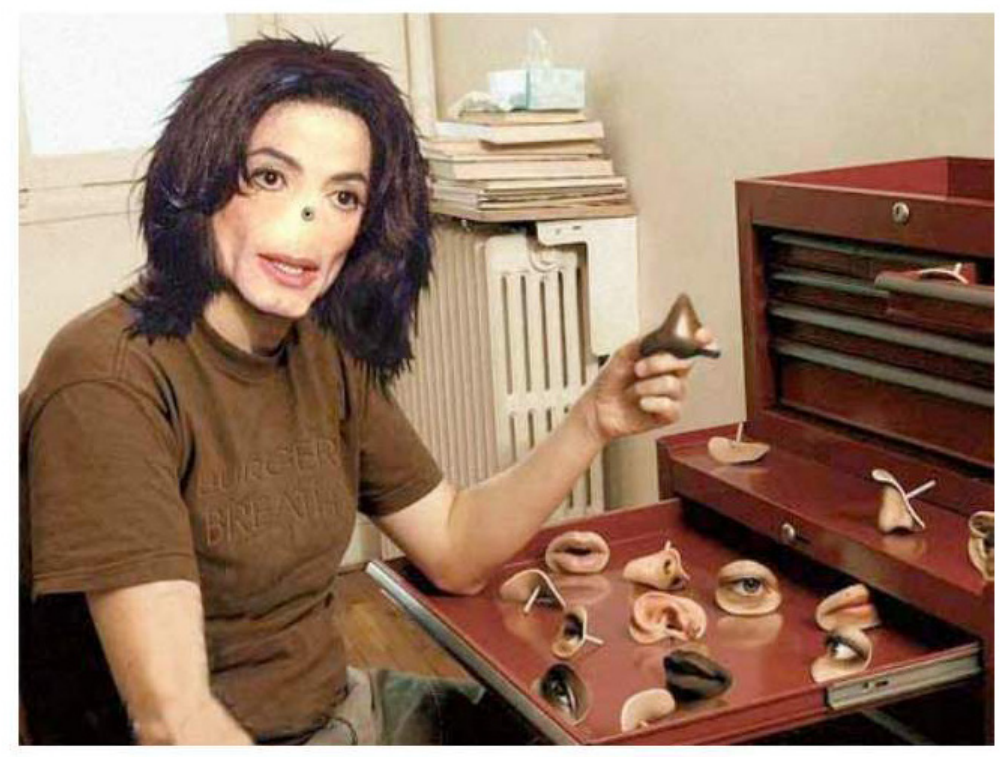

IMAGE 4 - Ce photomontage, présentant Michael Jackson comme Mister Potatobead, est une image anonyme circulant sur Internet. Elle est par exemple diffusée sur le site bloggits.com en 2008.

FIGURE 4

1 Jeu dans lequel chacun compose son propre Michael Jackson : www.alexanderband. $\mathrm{dk} / \mathrm{dragson} / \mathrm{index} \cdot \mathrm{htm}$.

2 www.bloggits.com/2008/7/28/Pictures/Funny/Micheal-Jackson-Potato-Head 
Ces représentations apparentent Michael Jackson à un enfant jouant avec son propre corps, changeant d'apparence comme de vêtement, au gré de ses désirs. Dès lors, son corps n'a plus rien d'humain : artificiel et malléable, il devient un support d'expression pour les fantasmes du chanteur... et de ceux de ses fans par la même occasion ! Mais simultanément, ce type d'imagerie accroît le sentiment « d'inquiétante étrangeté » (Freud, 1985) qu'inspire Michael Jackson à certains publics.

\section{Du non humain à l'inhumain : vers la monstruosité}

À l'issue de ce long processus de déshumanisation favorisé par les appropriations successives des publics et accéléré par la démocratisation d'Internet au cours des années 2000, Michael Jackson est bientôt entrevu comme une figure monstrueuse. Dérivant du latin monstrum, le monstre est celui que l'on montre. Comme le dit Régis Bertrand, «Dans les foires, le monstre qu'on exhibe est celui qui est plus grand, plus gros, plus petit, plus maigre, plus poilu que l'homme ordinaire [...]. Cette norme est, le plus souvent, de nature arithmétique : c'est une moyenne, qui situe le monstre du côté du corps extrême » (Bertrand \& Carol, 2005, p. 7). Or, c'est bien cette anormalité de Michael Jackson que les internautes veulent souligner, mon(s)trer, au sein de leur production. Le monstre également, représente l'altérité radicale par opposition à une normalité socialement reconnue. Or cette normalité, dont seules les sociétés fixent les règles, est assimilée au concept « d'immuable nature » (Burgos, 1975, p. 16) : autrement dit, dans l'esprit des hommes, le monstre qui sort de la norme "rompt l'ordre logique de l'univers " (Kappler, 1980, p. 52). Tel le monstre, le Michael Jackson des années 1995-2000 semble aller « contre nature ».

En effet, sa dimension monstrueuse commence à être évidente dès la fin de l'année 1993, lorsque le chanteur est accusé pour la première fois de pédophilie ${ }^{1}$. Wacko Jacko prend peu à peu le pas sur King of Pop et $B a m b i$, et les affects négatifs sont encouragés au dépend des modes d'adhésion positifs. L'imagerie produite par les internautes depuis le milieu des années 1990 montre en effet le chanteur comme un double dégénéré de lui-même. Par exemple, c'est à un clone raté de Superman

1 Le chanteur règle l'affaire par un arrangement financier. Cette transaction, mal perçue par les médias et l'opinion, encourage les soupçons à l'égard de sa culpabilité supposée. 
nommé Bizarro Superman que l'internaute Rahni compare le Michael Jackson de 1997. Bizarro Superman est l'anti-Superman, le double négatif, qui cherche à devenir " parfaitement imparfait » en agissant systématiquement dans le sens contraire des vertus humaines. De façon analogue, Wacko Jacko représente le double inversé du King of Pop.

Dans la même lignée, l'auteur de cette page, compare le Michael Jackson de 1999 à l'antagoniste du super héros Batman : le Joker. Le rapprochement est plus fort qu'il n'y paraît, si on se souvient que le Joker a été défiguré par l'acide et qu'il cache sa monstruosité derrière un épais maquillage de clown lui donnant un air inoffensif. Tout dans le personnage du Joker renvoie au monde de l'enfance : son univers acidulé, ses armes ressemblant à des jouets, son humour potache... De manière analogue, Michael Jackson était fasciné par les univers enfantins : il possédait un parc d'attraction, aimait la compagnie des enfants, les dessins animés... À la lumière des poursuites judiciaires pour pédophilie ${ }^{1}$ que le chanteur doit affronter en 2003, au moment où l'internaute produit cette page, cette comparaison laisse entendre que le chanteur se sert, comme le Joker, de jouets étincelants pour attirer d'innocentes victimes. Cette représentation, particulièrement emblématique, rend compte du fait qu'à partir de cette époque les détracteurs du chanteur l'envisagent ${ }^{2}$ comme un être déviant aux «pratiques inhumaines ». Dans leur esprit, sa hideur physique (toujours accentuée par les médias et les internautes) se veut être le témoin de la hideur morale qu'il cache.

Après avoir incarné les fantasmes narcissiques d'un large public et l'Idéal du Moi de nombreux fans, Michael Jackson est dorénavant le réceptacle des peurs inconscientes de certains : dans le contexte de

1 L'adolescent Gavin Arvizo accuse le chanteur d'attouchement sexuel en 2003. Cette affaire donne lieu à un procès en 2005, à l'issue duquel Michael Jackson est reconnu non coupable de tous les chefs d'accusation.

2 On trouve, dans les médias et sur Internet, de nombreuses représentations allant dans ce sens : par exemple, la couverture du pamphlet de Jean-Paul Bourre Bad: Michael Jackson, le Mutant (2004, éditions Bartillat) montre un Michael Jackson inquiétant plongé dans une semi pénombre (le livre est d'ailleurs un plaidoyer accusant le chanteur d'actes monstrueux). Un autre exemple célèbre est le clip vidéo du rappeur américain Eminem Just lose it sortit en 2004 qui représente Michael Jackson comme un pédophile (il s'amuse sur un lit avec des enfants) à l'allure décrépite (ses cheveux prennent feu et il perd son nez). 
« psychose pédophile » (Jenkins, 1998) qui règne en Occident dans les années 1990-2000, il catalyse les passions liées au ressurgissent de ce tabou ; affichant dorénavant un visage complètement transformé et systématiquement « zombifié » par les médias et les internautes, il figure dès lors le double obscur que certains refoulent en eux-mêmes.

\section{Michael Jackson : un symbole de la damnation}

À l'issue de ce long processus de co-construction, les grandes lignes narratives du mythe sont relativement établies et forment, conformément au modèle de Philippe Marion, un seul et même récit répondant à un modèle mythique ancestral : celui qui raconte la damnation d'un homme ayant voulu égaler Dieu.

Cette signification globale est évidente lorsqu'on observe les productions d'amateurs qui circulent sur Internet quelques années avant la mort du chanteur (entre 2003 et 2009). Un photomontage très emblématique montre par exemple Michael Jackson sous les traits de Mikem $^{1}$ (Figure 5) - une créature semblable au célèbre Gollum du Seigneur des Anneaux². Gollum, anciennement nommé Sméagol, n'a de cesse de reprendre possession d'un anneau qui lui apportera toute puissance et immortalité : aliéné à ce désir inaccessible, il se transmue en une créature monstrueuse dont la décrépitude physique traduit la déchéance mentale. Le parallèle est rapidement établi avec King of Pop : par ses transformations physiques et ses mises en scène, Michael Jackson semble en quête d'omnipotence et d'immortalité.

Or, dans la symbolique judéo-chrétienne, celui qui tente de se hisser au rang de Dieu est damné et la déchéance physique est le prix par lequel il « paie le crime suprême, celui d'impiété » (Wolff-Quenot, 1996, p. 155-156). Plus précisément, selon Marie-Josèphe Wolff-Quenot, « la métamorphose en monstre est vengeance d'un dieu courroucé, d'une fée malveillante, d'un sorcier malfaisant. C'est la punition ad aeternam d'une faute jugée inexpiable autrement » (Wolff-Quenot, 1996, p. 156). C'est le sort que subit Gollum dans sa quête de « l'anneau de puissance » : aliéné à son désir d'omnipotence, hanté jours et nuits par des puissances diaboliques (il entend des voix), il devient malingre,

1 www.guzer.com/pictures/golem_jackson.php

2 Roman en trois volumes de J. R. R. Tolkien paru en 1954 et 1955. 
perd ses cheveux, et sa peau se flétrit. C'est aussi le sort que subit l'ange Lucifer, puni pour avoir voulu s'affranchir de la loi suprême, et dont l'iconographie insiste souvent sur la décrépitude du corps : ailes décharnées, teint cadavérique... À leur image, Michael Jackson devenu surhomme, ne pouvait que s'exposer à Némésis, la vengeance divine qui, depuis toujours, punit l'homme de son orgueil.

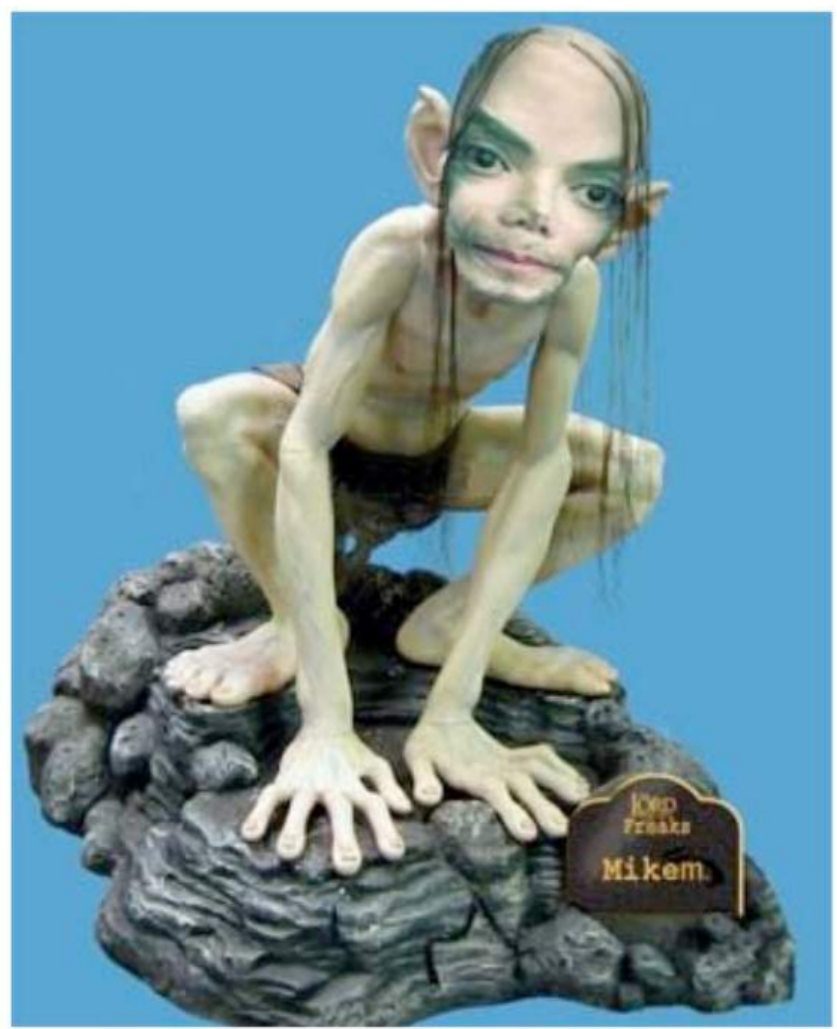

IMAGE 5 - Photomontage anonyme, circulant sur Internet, et représentant Michael Jackson sous les traits de «Mikem », personnage monstrucux empruntant au fameux Gollom du Seigneur des anneanx,

FIGURE 5 
À compter des années 2002-2003, de nombreuses représentations d'amateurs alimentent ce type d'imagerie et on trouve même sur des forums quelques discussions d'internautes prétendant que la star aurait pactisé avec le diable. Dans l'esprit du public, Michael Jackson est devenu « pour de vrai » le zombie qu'il incarnait « pour de faux » dans Thriller. Les comparaisons de l'internaute Rahni en sont encore la parfaite illustration (Figure 6) : le Michael Jackson de 2002 est présenté comme le monstre du film d'horreur Creature from the Black Lagoon ${ }^{1}$, ou encore comme le revenant d'outre-tombe d'Evil Dead'. À partir des années 2000, il n'est pas rare d'observer ce type de représentations dans les médias et particulièrement sur Internet : un épisode de la série animée américaine Hollywood Zombies ${ }^{3}$ montre par exemple le chanteur tel un mort vivant (des vers lui mangent la peau) ou encore, un masque (le Wacko Jacko Mask ${ }^{4}$ ) présentant Michael Jackson tel Frankenstein est commercialisé sur les sites de déguisement après 2005.

Or le mort-vivant symbolise la possession, l'aliénation mentale d'un individu condamné à être dépossédé de lui-même et asservi à une volonté extérieure. Pour Pierre Brunel, il est symbole d'une « mise en garde contre tout ce qui [...] en veut à notre liberté » (Brunel, 1999, p. 897), contre tout ce qui nous aliène, et notamment contre l'illusion séductrice qui nous détourne de nous-mêmes, et qui nous est parfois offerte par quelques figures idéalisées. Le zombie finalement, est une figure mythique qui se révèle être « moins un mythe qu'un anti-mythe » (Brunel, 1999, p. 897) et le mythe de Michael Jackson, comme tous les grands récits, délivre aux publics une vérité essentielle : à l'instar de Lucifer, Faust ou Frankenstein, il met en garde l'homme contre l'aliénation narcissique et le refus de sa condition.

1 Film américain de Jack Arnold (1954). Une créature préhistorique est découverte par un scientifique au fond d'un lagon.

2 Film de zombies américain de Sam Raimi (1981).

3 Cette vidéo produite et publiée par le site Hollywoodzombies.com en 2007 (disparue depuis) est toujours visible en 2012 sur YouTube, Dailymotion ou Veoh.

4 Proposé notamment sur le site imockery.com à l'adresse suivante : http://www.imockery.com/halloween/bag/wacko-jacko.php 


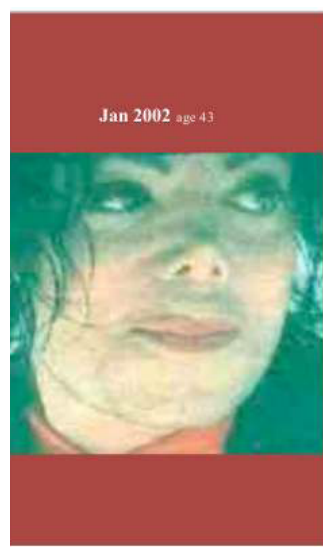

- The "Black Lagoon" phase

Big news brings Michael out of his Howard Hughes-like life and back into the spotlight when he charges his record company, Sony, is "racist". That's why his "Invincible" CD sold 2 million copies; Sony didn't promote him enough. He sez. We all get to gasp anew and ask the question - WTF?! Seems he's had his nose fixed,.,WHEW! and just got out of bed. As it is reported in the news it seems a bridge was built to widen the nasal passages. "Thank God!" the headlines say. Poor thing probably couldn't breathe! with those teensy bitty nostrils. Oh How Nice For Him! Perhaps his singing will improve, since his last album was 70 minutes of hiccups, grunts, fake crying and yips. One has to wonder why, with all his money, he can't seem to find plastic surgeons who are capable of actually doing plastic surgery. The "fixed" bridge appears as two lumpy lines and not what say, just for the sake of argument, a plastic surgeon might put in someone's face to create a nose bridge. Maybe this is a new trend in Breath-Rite Strip implants? One wonders what those Jutting Gill Bumps were on the sides of his jaw in 1997. Mike ruins his symmetry schtick with mismatched, lopsided eyes and lipstick like my senile Aunt Margaret wears. Music critics and even those in the record industry are saying $\mathrm{OK}$, quite enough from this goof. There is even a TV special in the UK asking - If this is what the guy is doing to his outside, then what the hell is going on inside? What's happened to our Michael?

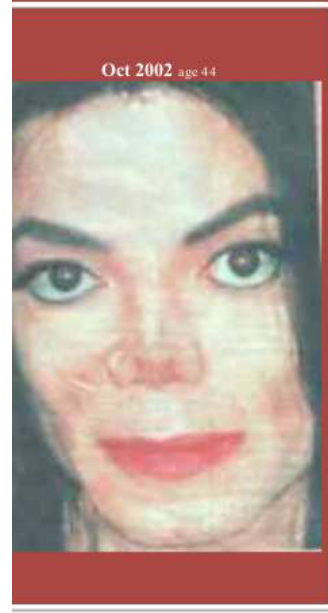

Tracey Orvez took this photo in the parking lot of the Beverly Regent Hotel in Beverly Hills, Califomia. She heard he was there so waited in hopes of seeing him. What a surprize it must have been to see The Mike, making his way to his limo dressed in only blue Jammies with snow flakes and polar bears on them. Always a good look for a star, I say. She asked if she could take his photo and he said sure... as long as she "stood well back".

I can't imagine why

The publicly decried "third nostril", which appeared after the January plastic surgery (see above photo) that a few took time out of their busy days to write and inform me I was full of shit about, seems to be closing up but has left an obvious scar. The tip, which has been rumored (damn, I'm good) to have died and/or be a puttied-on prothesis looks to be dead tissue and/or a puttied on prothesis. Said Ms. Orvez: "He looked like a ghoul. When I had the picture developed, I was sick. The guy doesn't appear to have a nose."

Well, when you hack away at it for 15 years, that happens. But as my detractors write to me, I just put these vicious "lies" up because I'm "jealous". I am, boy howdy! I'm jealous I don't have whatever it is Mike's on to make his pupils the size of dinner plates. And I would Mike's on to make his pupils the size of dinner plates. And I would
really love some Polar bear and snowflake jammies myself. I wish Tracey mentioned if they had Feets in them or not! It's hell trying to find "fun" jammie feets pajamas when you're a grown up woman. How envious I am that a grown man can!

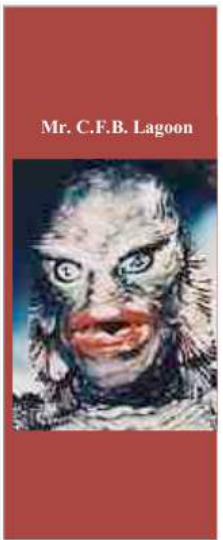

IMAGE 6 - Capture de la page web A Photographic History of Michael Jackson's Face

FIGURE 6

\section{Mythologie post-mortem et transmission}

Enfin, après avoir participé activement à la co-construction de la croyance en Michael Jackson, les internautes contribuent aussi largement, depuis sa mort, à la pérennisation de son mythe et à l'institutionnalisation de son culte. Par l'intermédiaire de vidéos postées sur Youtube, de forums, de sites Web ou de groupes constitués sur les réseaux sociaux, ils écrivent une nouvelle page de l'histoire du chanteur et tentent de rationaliser la croyance en Michael Jackson. 
Certains internautes, à l'instar des journalistes, continuent de colporter toutes sortes de rumeurs farfelues. On dit ainsi que Michael Jackson souffrait de nombreuses maladies : cancer de la peau, maladie génétique, problèmes respiratoires, nécrose du nez... Quelques jours après sa mort, on affirme aussi jusque dans les grands médias ${ }^{1}$ qu'il se ferait embaumer dans la position du Moonwalk ${ }^{2}$ aux côtés de son chimpanzé. De même, les détracteurs du chanteur s'évertuent encore à le discréditer, à l'instar des membres du groupe Facebook français Anti Michael Jackson ${ }^{3}$. Ces récits permettent au personnage Wacko Jacko de survivre à la mort du chanteur et, pour les plus prosélytes, de combattre les représentations positives diffusées par les amateurs fidèles et engagés du King of Pop.

Du côté des partisans justement, une véritable campagne de réhabilitation du chanteur est engagée sur Internet. Les fans-clubs du monde entier prescrivent des manières de croire aux nouveaux adeptes, en élaborant toutes sortes de dossiers et enquêtes sur le chanteur, en animant multitude de forums, en indiquant les ouvrages incontournables consacrés à l'artiste ou, au contraire, en menant des vendettas contre ceux qu'ils méprisent, en organisant toutes sortes de commémorations, ou encore en diffusant massivement des messages de solidarité sur les réseaux sociaux. Les fans-clubs officiels, menés par le Michael Jackson Estate ${ }^{4}$, procèdent ainsi à une véritable institutionnalisation de la croyance en Michael Jackson.

Cependant, ces initiatives ne se font pas sans dissensions et de nombreux groupuscules marginaux et prosélytes apparaissent, promouvant chacun des modes d'adhésion et des croyances spécifiques. La communauté des This is Not It (TINI) $^{5}$ par exemple dénonce une conspiration : selon eux, l'industrie et les promoteurs de concerts auraient jugés Michael Jackson plus rentable mort que vivant. Chaque jour, les membres de ce collectif sensibilisent de nouveaux fans à leurs théories, diffusant des pétitions, créant des pages ou des vidéos sur

1 Information relayée en France par BFM Télé.

2 Célèbre pas de danse du chanteur.

3 www.facebook.com/group.php?gid=94225852347\&ref=search

4 Groupe officiel américain composé des exécuteurs testamentaires du chanteur, dirigé par l'avocat J. Branca et le producteur J. McClain.

5 www.this-is-not-it.com 
les réseaux sociaux, et livrant même une guerre acharnée à tous les incroyants. À leurs yeux, Michael Jackson est avant tout une victime naïve prise au piège des intentions malfaisantes de son entourage. Ils perpétuent ainsi la mémoire du fameux Bambi.

À l'opposé, ceux qui croient en la toute puissance du King of Pop refusent d'admettre la disparition du héros. Sur Internet, ils prétendent à une supercherie : « Michael Jackson a mis en scène sa propre mort ! », affirment-ils, preuves à l'appui ${ }^{1}$. À leurs yeux, les images de $C N N^{2}$ tournées pendant le transport du corps en hélicoptère de l'hôpital au centre médico-légal, font apparaître un mouvement de la dépouille. De même, confortés par la retenue apparente de la famille du chanteur au cours de ses obsèques ou lors de leurs interventions télévisées, ces internautes en déduisent que le décès du chanteur est un coup monté. Selon eux, Michael Jackson aurait même fait plusieurs apparitions publiques depuis sa mort, caché derrière des maquillages élaborés. Ils disent ainsi que la star aurait assisté à son propre enterrement, sous l'apparence d'un mystérieux homme à chapeau ou d'une jeune femme blonde ${ }^{3}$. Ils prétendent aussi que la star se serait exprimée à la télévision américaine sous les traits du docteur Conrad Murray ${ }^{4}$, dont les autorités suspectent pourtant l'implication dans la tragédie, ou de Dave Dave ${ }^{5}$, un grand brûlé ami de Michael Jackson. Les spéculations de ces fans, nommés les beLIEvers ${ }^{6}$ (ceux qui croient en un mensonge - «lie » en anglais), continuent à construire la dimension surhumaine du chanteur : elles dépeignent un Michael Jackson tout puissant et immortel capable d'organiser le plus gros coup médiatique de l'histoire.

1 Par exemple, la chaine Youtube de l'internaute canadienne Julia142, consacrée à la production et à la diffusion de vidéos voulant prouver que la star n'est pas morte : www.youtube.com/user/julia142

$2 C N N, 25$ juin 2009.

3 La vidéo Hat Man At Michael Jackson Memorial And Funeral. Who Is He?!?!, publiée le 19 octobre 2009 sur Youtube.

4 La vidéo Michael Jackson Is Alive New Exclusive Evidence, postée sur Youtube par l'utilisateur xikzfonehome, le 17 octobre 2009.

5 La vidéo Michael Jackson Is ALIVE, Here His Last Disguises!, diffusée par l'utilisateur alwaysbelieveMJ sur Youtube, le 16 décembre 2009.

6 Terme provenant du spectacle beLIEve du magicien américain Criss Angel, que les internautes croient impliqué dans le hoax. Cf. par exemple la vidéo de amel82light, The Michael Jackson Death Hoax: Criss Angel Proofs, postée le 4 mars 2010. 
Aujourd'hui, c'est ainsi une guerre d'influence que se livrent les différents groupuscules de la communauté « jacksonienne ». Les schismes latents qui divisaient les croyants sont plus que jamais avivés. Et face à l'arrivée massive de nouveaux fans, les anciens se replient pour tenter de faire le tri entre ceux qu'ils nomment les « vrais » et les « faux fans ». Ils instaurent des codes et des référentiels toujours plus précis pour se distinguer ${ }^{1}$, ainsi que des rituels pour raviver leur sentiment d'appartenance : chaque année par exemple, l'anniversaire du chanteur (comme celui de sa mort) est l'occasion pour différents groupes de fans d'organiser des rassemblements, hommages, flash-mob ${ }^{2}$ (pour « mobilisation flash ») ou pèlerinage (à Eurodisney, au cimetière Forest Lawn où il est enterré, aux anciennes demeures ${ }^{3}$ du chanteur). Chacun tente d'institutionnaliser la croyance et le culte en revendiquant la légitimité de ses conceptions, en recrutant de nouveaux croyants ou en chassant les dissidents. Face aux thèses conspirationnistes par exemple, fans-clubs officiels et fans modérés luttent sans merci : ils bannissent de leurs sites et forums tous ceux qui osent promouvoir de telles idées, instaurant par là-même des sujets tabous et des modalités de croyance hérétiques. Michael Jackson est à leurs yeux un véritable prophète contemporain.

\section{Conclusion}

Véritable objet de croyance, tant de son vivant qu'après sa mort, Michael Jackson est le réceptacle d'affects puissants et contrastés : à la fois figure charismatique qui fascine et séduit et figure monstrueuse qui effraye et inquiète, il invite les différents publics à projeter sur lui une part de leur imaginaire. Appropriable à loisir, ce personnage s'est rapidement construit comme un mythe : engendré par de multiples acteurs, il a appelé une participation active des médias, des industries et des publics, qui ont conjointement façonnés ses contours et encouragé la croyance en sa mythologie.

1 La communauté des fans qui revendiquent que justice soit rendue au chanteur (le collectif Justice For Michael) arbore par exemple toute sorte de tee-shirts, pancartes, badges ou avatars reprenant couleurs, logotype ou initiales du collectif.

2 Cf. par exemple le collectif Thrill the world qui organise chaque année des mobilisations flash simultanées dans le monde entier, au cours desquelles les fans interprètent une chorégraphie du chanteur. (thrilltheworld.com).

3 À Gary dans l'Indiana, où le chanteur a passé son enfance par exemple. 
Cette figure planétaire qui s'est construite durant plus de quarante années en impliquant les publics du monde entier, révèle les transformations fulgurante du monde "médiaticoculturel »: internationalisation de la culture, globalisation des réseaux de communication, explosion de l'offre mass-médiatique, démocratisation d'Internet, passage de la starification à la peoplisation, appropriation massive du numérique, fragmentation des publics et des médias et formation d'agrégats communautaires correspondants, apparition et développement croissant d'une culture participative... Figure polymorphe et mouvante qui voit encore aujourd'hui ses contours évoluer, Michael Jackson est à l'image de la modernité qui l'a engendré.

Internet a servi de caisse de résonnance aux phénomènes d'appropriation collective qui ont co-construit son personnage public et la croyance qu'il suscite encore aujourd'hui. Grâce aux empreintes que laissent les internautes lors de leurs appropriations successives, il est dorénavant possible de retracer le parcours d'une icône en cours de construction et de redonner aux publics la place centrale qu'ils occupent dans l'élaboration des récits qu'ils consomment et des croyances qu'ils investissent.

\section{Références}

Assoun, P.-L. (1990). L'école de Francfort, Paris : Presses universitaires de France.

Bertrand, R., \& Carol, A. (Éd.). (2005). Le Monstre humain. Imaginaire et Société, Aixen-Provence : Publications de l'université de Provence.

Brunel, P. (Éd.). (1999). Dictionnaire des mythes d'aujourd'hui. (pp. 889-898). Monaco : Éditions du Rocher.

Burgos, J. (Éd.). (1975). Cahiers de recherche sur l'imaginaire : le monstre - 1. Présence du monstre. Mythe et Réalité. Paris : Lettres modernes.

Camilleri, C. et al. (1997). Stratégies identitaires. Paris : Presses universitaires de France.

Certeau, M. (de) (1987). La faiblesse de croire, Paris : Seuil.

Dalmazzo, A. (2009). Charismes, Identités, Fanatismes. Le charisme médiatique et les fans de Michael Jackson. L'Idéal et le Monstre. Dissertation doctorale, Sciences de l'information et de la communication, Université Paris II.

Dorna, A. (1998). Le Leader charismatique. Paris : Desclée de Brouwer. 
Freud, S. (1918). Tabou sur la virginité. Dans A. Bourguignon \& P. Cotet (Éd.), Oeuvres Complètes. Pychanalyse, t. XV. (pp. 77-96). Paris : Presses universitaires de France. Freud, S. et al. (1997). L'identification : L'autre, c'est moi. Paris : Tchou.

Freud, S. (1985). L'inquiétante étrangeté et autres essais. (pp. 208-263). Paris : Gallimard.

Jenkins, P. (1998). Moral Panic: Changing Concepts of the Child Molester. Dans Modern America, New Heaven (CT) : Yale University Press.

Neuilly, M.-A., \& Zgoba, K. (2005). La panique pédophile aux États-Unis et en France. Champ pénal/Penal field [En ligne], XXXIVe Congrès français de criminologie. Disponible à : http://champpenal.revues.org/340.

Kaës, R. (1998). L'appareil psychique groupal. Constructions du groupe. Paris : Dunod.

Kappler, C. (1980). Le Monstre. Pouvoirs de l'imposture. Paris : Presses universitaires de France.

Kristeva, J. (1997). Au commencement était l'amour : psychanalyse et foi. Paris : Librairie générale française.

Laplanche, J., \& Pontalis, J.-B. (2002). Vocabulaire de la psychanalyse. Paris : Presses universitaires de France.

Le Bart, C. (2000). Les fans des Beatles, sociologie d'une passion. Rennes : Presses universitaires de Rennes.

Le Guern, P. (Éd.). (2002). Les cultes médiatiques. Culture fan et œuvres cultes. Rennes : Presses universitaires de Rennes.

Maigret, E., \& Macé, E. (2005). Penser les médiacultures. Nouvelles pratiques et nouvelles approches de la représentation du monde. Paris : Armand Colin.

Marion, Ph. (1997). Narratologie médiatique et médiagénie des récits, Recherches en Communication, 7, 61-87.

Mijolla-Mellor, S. (de) (2004). Le besoin de croire : métapsychologie du fait religieux. Paris : Dunod.

Saussure, T. (de) (Éd.). (1996). Les miroirs du fanatisme. Genève : Labor \& Fidès.

Segré, G. (2003). Le culte Priestley. Paris : Presses universitaires de France.

Tisseron, S. (1997). Psychanalyse de l'image : des premiers traits au virtuel. Paris : Dunod.

Weber, M. (2006). Sociologie des religions. Paris : Gallimard.

Wolff-Quenot, M.-J. (1996). Des monstres aux mythes. Paris : Guy Trédaniel Éditeur. 